\title{
Targeting the Human Epidermal Growth Factor Receptors with Immuno-PET: Imaging Biomarkers from Bench to Bedside
}

\author{
Gabriela Kramer-Marek ${ }^{1}$ and Wim J.G. Oyen ${ }^{1,2}$ \\ ${ }^{1}$ Division of Radiotherapy and Imaging, Institute of Cancer Research, London, United Kingdom; and ${ }^{2}$ Royal Marsden NHS \\ Foundation Trust, Department of Nuclear Medicine, London, United Kingdom
}

Human epidermal growth factor receptors (HERs) are targeted by a growing number of inhibitors that directly block the receptors on HER-expressing tumor cells or interfere with their signaling pathways. However, HER expression is variable between tumors, and resistance against these drugs is a well-known clinical problem necessitating further research to optimize therapeutic regimes for HER-positive patients. Currently, information about the potential biomarker status is routinely obtained ex vivo from biopsy specimens. HER-targeted imaging biomarkers, on the other hand, could measure the receptor expression in vivo across the entire disease burden and help to monitor early responses to treatment. This review describes the current status of HER-specific imaging agents, with a particular focus on moving these molecular probes from early preclinical studies to clinical trials.

\section{EPIDERMAL GROWTH FACTOR RECEPTORS (EGFRs) AS VALID IMAGING TARGETS}

The HER family of receptor tyrosine kinases has been the focus of intense translational research over the past 3 decades. The HER family includes 4 members: EGFR (HER1, ErbB1), HER2 (ErbB2), HER3 (ErbB3), and HER4 (ErbB4). These structurally related proteins coordinate a complex signaling network that plays a crucial role in the development and evolution of cancer (Fig.1). There are at least 11 different ligands (e.g., epidermal growth factor, transforming growth factor, and neuregulins [NRGs]) that are known to bind to these receptors. Although all 4 receptors have the same essential domains, the functional activity of each domain varies. HER2 does not have a known ligand but exists in the extended "active" conformation state, constitutively available for dimerization with the other 3 members. In contrast, HER3 can bind to several ligands but lacks fully intrinsic tyrosine kinase activity because it is unable to bind adenosine triphosphate. EGFR and HER4 have active tyrosine kinase

\footnotetext{
Received Mar. 13, 2016; revision accepted May 11, 2016.

For correspondence or reprints contact: Gabriela Kramer-Marek, Institute of Cancer Research, Centre for Cancer Imaging, 15 Cotswold Rd., Sutton, Surrey SM2 5NG, U.K

E-mail: gabriela.kramer-marek@icr.ac.uk

Published online May 12, 2016.

COPYRIGHT (c) 2016 by the Society of Nuclear Medicine and Molecular Imaging, Inc.

DOI: $10.2967 /$ jnumed.115.169540
}

domains and known ligands (1). Ligand binding triggers intracellular signaling through a tightly controlled network of downstream signaling mechanisms that drive and regulate many cellular processes. The two main signaling pathways, which are used by all HER receptors, are ras protein/ mitogen-activated protein kinase, which leads to cellular proliferation, differentiation, and migration, and phosphatidylinositol 3-kinase/protein kinase B (PI3K/Akt), which primarily serves to promote cell proliferation and survival through progression of the cell cycle and inhibition of apoptosis (2). Changes in the regulation of receptor activity may cause uncontrolled cell growth and result in the initiation and maintenance of several solid tumors, including breast, gastric, ovarian, and non-small cell lung. Cancer patients whose tumors have HER alterations (e.g., receptor overexpression, mutation, or gene amplification) tend to have more aggressive tumor behavior. Furthermore, in most cases receptor positivity is associated with resistance to certain types of chemotherapy, hormone therapy, and ionizing radiation, consequently leading to a shorter time to disease progression and worse patient survival (2).

These findings have led to the development and widespread implementation of specific HER inhibitors, including antibodies targeting the extracellular domain and small molecules that prevent phosphorylation of the intracellular domain (tyrosine kinase inhibitors). Several of these targeted drugs have been approved for clinical use, including a recombinant humanized version of an anti-HER2 monoclonal antibody (mAb), trastuzumab (Herceptin), which has shown significant clinical efficacy in HER2-overexpressing metastatic breast cancers when combined with chemotherapy. The success of trastuzumab was followed by the introduction of other therapeutic antibodies and antibody-drug conjugates targeting HER2, such as pertuzumab (Perjeta; Genentech) and ado-trastuzumab emtansine (Kadcyla; Genentech). Among the small-molecule inhibitors, lapatinib (Tykerb; Novartis) has shown activity in HER2-positive cancers that have relapsed after trastuzumab treatment. Gefinitib (Iressa; AstraZeneca) and erlotinib (Tarceva; Genentech) are further examples of tyrosine kinase inhibitors, which act on the EGFR. The chimeric anti-EGFR mAb cetuximab (Erbitux; Lilly) is used for the treatment of metastatic colorectal cancer, metastatic non-small cell lung cancer, and head and neck cancer. 


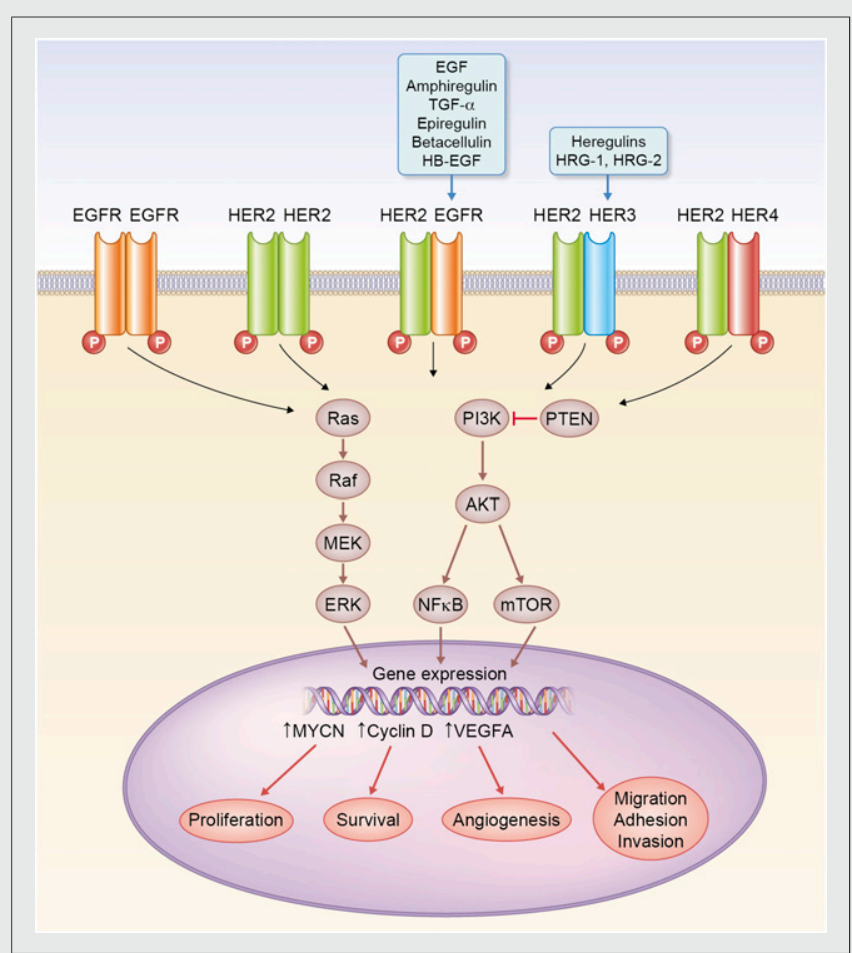

FIGURE 1. EGFRs and their major activation pathways. HB-EGF = heparin-binding EGF-like growth factor; $N F_{\kappa} B$ = nuclear factor- $\mathrm{B}$; mTOR $=$ mammalian target of rapamycin; TGF- $\alpha=$ transforming growth factor $\alpha$; VEGFA = vascular endothelial growth factor $\mathrm{A}$.

Even though these targeted therapies have improved the treatment outcome in the early stage of cancer, many tumors still manifest intrinsic resistance and even those that initially respond to the treatment develop resistance within a year.

The receptor status thus represents a major factor in selecting patients for targeted therapies; therefore, more precise assessment of the HER family expression levels and activation of downstream signaling pathways would prove beneficial in identifying new treatment paradigms.

Currently, HER-specific alterations (e.g., protein overexpression, receptor mutation, translocation, and epigenetics changes) can be analyzed by methods such as immunohistochemistry, quantitative polymerase chain reaction, proteomics, and next-generation sequencing of the tumor tissues derived postoperatively or via biopsies. These techniques aid in our understanding of how cancer cells adapt to the treatment and become resistant to it. However, these are invasive methods prone to sampling errors and most likely confounded by interand intratumor heterogeneity of receptor expression within analyzed biopsy specimens. Moreover, collecting sequential biopsies to monitor changes in receptor expression over time in response to treatment not only is practically difficult and ethically challenging but also does not reflect the presence of the resistant clones in multiple metastatic sites. Therefore, developing and validating noninvasive specific imaging biomarkers capable of confirming the presence of accessible targets early in the disease progression may help to resolve the dilemma that occurs when a new treatment regimen is being considered and information about protein status is essential but biopsy impractical.

PET using radiolabeled mAbs, antibody fragments, or engineered protein scaffolds (immuno-PET) has the potential to offer noninvasive criteria able to identify the presence and accessibility of the target, accurately measure tumor response immediately after treatment initiation, and assist in patient stratification. Furthermore, immuno-PET can provide information about the heterogeneity of both target expression and therapeutic response, which are increasingly recognized as key factors in therapeutic resistance, especially in patients with advanced disease for which target expression may vary from site to site and biopsy of a single site may not represent the entire burden of a disease. The noninvasive nature of immunoPET also allows for performing multiple imaging sessions to adequately assess treatment response by longitudinal studies.

Although introduction of immuno-PET into routine clinical practice may add some complexity and increase the costs of studies, with appropriate use it has the potential to assess the efficacy of novel anticancer compounds during the early stages of development. It will also minimize the investment wasted by taking ineffective treatments through further clinical testing. Noteworthy, in 2012 Pfizer announced that $43 \%$ of its phase II trials failed because of a negative outcome from clinical proof-of-concept studies for which the candidatedrug mechanism of action was not adequately tested in an earlier phase of development (3).

\section{ROLE OF IMAGING HER RECEPTORS IN PATIENT STRATIFICATION AND MONITORING THERAPY RESPONSE}

With the increasing number of therapeutic antibody candidates targeting members of the HER family, there is a need to better understand their biochemical and biologic properties. Recently, several research groups have intensively worked on the development of imaging probes targeting these receptors, since the information about their expression helps to identify those patients who are likely to benefit from antiHER-targeted therapies. However, because only a few studies have explored the relationship between imaging, genomics, and histopathology, there is still a need for larger prospective studies to elucidate whether these methods can play a complementary role in assessing target expression and whether their combination may prove useful.

A growing spectrum of radionuclides with a longer half-life has recently become available for conjugation with mAbs. The most frequently used are ${ }^{124} \mathrm{I}$ (half-life, $4.17 \mathrm{~d}$ ), ${ }^{64} \mathrm{Cu}(12.7 \mathrm{~h}$ ), and ${ }^{89} \mathrm{Zr}(78.4 \mathrm{~h})$. Depending on the radioisotope, different labeling methods are used. Iodine can be directly attached to a $\mathrm{mAb}$ through the simple and widely available procedures, whereas radiometals are introduced indirectly by first conjugating the suitable chelator to the mAb and then noncovalently binding the metal ion. Among the radiometals, ${ }^{89} \mathrm{Zr}$ is the isotope of choice for $\mathrm{mAbs}$ that become internalized on binding to their target since it becomes trapped (residualized) 
inside the cell and its physical half-life matches well the relatively slow pharmacokinetics of mAbs in solid tumors.

Furthermore, ${ }^{89} \mathrm{Zr}$ can be chelated by desferoxamine under mild conditions that do not affect mAb structure. But slow clearance of intact mAbs requires a prolonged interval from injection to scanning, and one of the principal concerns regarding the use of ${ }^{89} \mathrm{Zr}$ in the clinic is the substantial radiation dose received by patients compared with shorter-lived nuclides. Indeed, it has been reported, for example, that ${ }^{89} \mathrm{Zr}$-trastuzumab (37 $\mathrm{MBq} ; 18 \mathrm{mSv}$ ) leads to a 2.5 -fold higher radiation exposure than conventional ${ }^{18} \mathrm{~F}-\mathrm{FDG}$ PET (37 MBq, $\left.\sim 7 \mathrm{mSv}\right)(4)$. These considerations have prompted the development of alternative approaches such as engineered $\mathrm{mAb}$ fragments, diabodies, and Affibody molecules that still have the high specificity and affinity of the potential therapeutic mAb but a molecular weight below $60 \mathrm{kDa}$ that allows for rapid renal clearance and elimination. The clearance time of these targeting agents also matches the favorable short half-life of ${ }^{18} \mathrm{~F}$ (109.8 min), allowing for same-day imaging.

Many preclinical small-animal studies have been performed with ${ }^{89} \mathrm{Zr}$-labeled anti-HER radioligands to determine their tumor-targeting characteristics. Knowledge obtained from these studies has enabled the translation of these agents into clinical trials. A typical example of such practice is the breast cancer studies that developed HER2-targeted therapies, which were one of the most significant breakthroughs in the treatment of HER2-positive patients and led to a marked increase in therapeutic response. However, because of disease heterogeneity, the reported discordance in HER2 status between primary tumor and metastasis as measured by immunohistochemistry has been high $(13 \%-30 \%)$, indicating the need to introduce immuno-PET imaging into clinical practice. Figure 2 shows a typical example of ${ }^{89} \mathrm{Zr}$-labeled trastuzumab PET/ $\mathrm{CT}$ in a patient with metastatic breast cancer.

An interesting early example is a study by Dijkers et al. on HER2-positive breast cancer patients (4). The authors demonstrated high accumulation of ${ }^{89} \mathrm{Zr}$-trastuzumab in HER2positive lesions in the liver, lung, and bone, and unknown metastases were detected across a locally compromised bloodbrain barrier. ${ }^{64} \mathrm{Cu}$-DOTA-trastuzumab has also shown promise in early clinical trials (5). In another study, Smith-Jones et al. showed that ${ }^{68} \mathrm{Ga}$-labeled Fab fragments detect different levels of HER2 receptors in preclinical models (6), and this approach has already demonstrated its feasibility in an early clinical study (7). Preclinical imaging using ${ }^{89} \mathrm{Zr}$-trastuzumab has also been used to monitor a decrease in HER2 expression in response to drugs targeting heat shock protein 90, a chaperone protein that plays an important role in mediating HER2 expression. Interestingly, these studies have recently been translated to early-stage human trials, in which ${ }^{89} \mathrm{Zr}$-trastuzumab imaging was used to measure changes in HER2 expression in response to NVP-AUY922, an inhibitor of heat shock protein 90 (8). Furthermore, it is well known that for ado-trastuzumab emtansine to be active, the presence of an intact HER2 receptor is of pivotal importance since internalization of the cytotoxic moiety of the drug depends on binding of trastuzumab to the

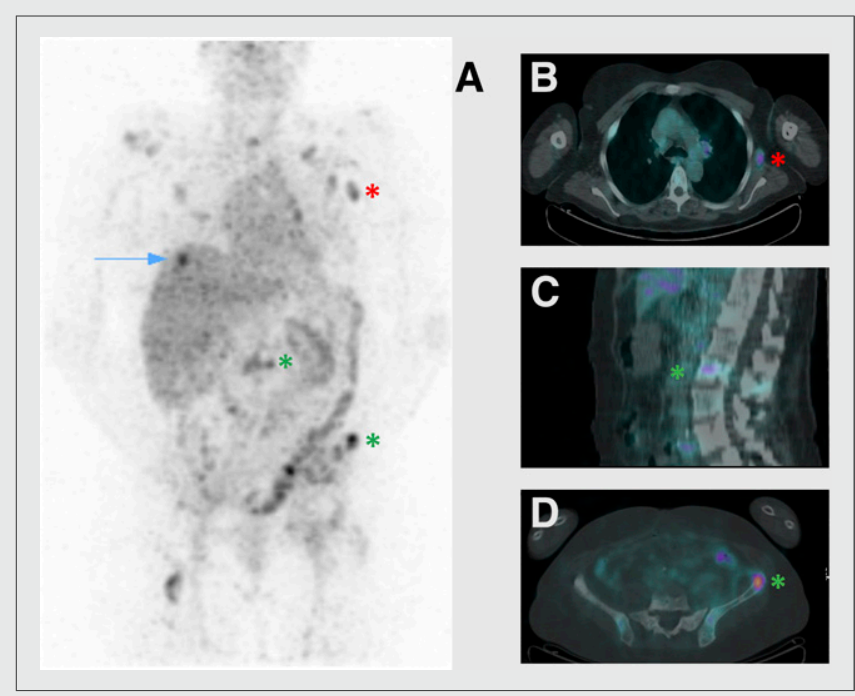

FIGURE 2. Whole-body PET (A) and cross-sectional PET/CT (B-D) images $96 \mathrm{~h}$ after intravenous injection of $37 \mathrm{MBq}$ of ${ }^{89} \mathrm{Zr}$-trastuzumab in patient with HER2-postive breast cancer metastatic to liver (arrow), lymph nodes (red asterisks), and bone (green asterisks). (Courtesy of Dr. Udai Banerji, Institute of Cancer Research, London, U.K.)

external domain of HER2. Gebhart et al., at the 2014 American Society of Clinical Oncology meeting, presented data showing that the presence or absence of HER2, as indicated by ${ }^{89} \mathrm{Zr}$-trastuzumab uptake, and response, as indicated by a decrease in ${ }^{18} \mathrm{~F}$-FDG uptake, provided an essentially $100 \%$ accurate prediction of later clinical response to HER2-directed therapy with ado-trastuzumab emtansine administration in metastatic HER2-positive breast cancer (NCT01565200 clinical trial) (9). This trial is a great example indicating that ${ }^{89} \mathrm{Zr}$-trastuzumab is an accurate noninvasive test capable of identifying nonresponding patients before ado-trastuzumab emtansine administration. Recently, several groups, including ours, have also tested the feasibility of using Affibody molecules specifically targeting HER receptors (10). In 2010, the first clinical studies using ${ }^{68} \mathrm{Ga}$ - and ${ }^{111}$ In-labeled Affibody-based HER2-imaging agents were performed (DOTA- $Z_{\text {HER2:342-pep2 }}$ [ABY-002] and ABY-025). Both compounds showed specific tumor uptake and allowed for highcontrast imaging of HER2-positive tissues $(11,12)$. Combined, these results further strengthen the applicability and promising future of HER2 imaging as routine clinical practice.

The ${ }^{89} \mathrm{Zr}$-labeled anti-EGFR mAb cetuximab has also been investigated, but most studies to date have been performed on animal models. Several groups have shown that receptor expression level alone is not sufficient to predict patient response to anti-EGFR therapies and that there is no correlation between tracer uptake and protein level as evaluated by immunohistochemistry (13). Other studies have demonstrated that most tumors responding to EGFR kinase inhibitors harbor activating mutations in the EGFR kinase domain (14), suggesting that imaging of EGFR mutant expression could be more useful in selecting the right patient population for personalized treatment. On the other hand, Chang et al. have recently shown that the 
uptake of EGFR-targeted ${ }^{89} \mathrm{Zr}$-panitumumab correlated well with the EGFR expression (15), and van Dijk et al. have shown that ${ }^{111}$ In-cetuximab-F $\left(\mathrm{ab}^{\prime}\right)_{2}$ can be used to monitor the effects of EGFR inhibition combined with irradiation in models of head and neck carcinoma (16). Also, comprehensive reviews on PET imaging of EGFR expression and multimodality imaging of the HER-kinase axis in cancer have been published $(17,18)$. Similarly to HER2 and EGFR PET, the very recent preclinical and clinical data emphasize the potential for HER3 imaging. This receptor is the only member of the family that lacks fully catalytic kinase activity, and accordingly its activation after heregulin binding is inherently dependent on heterodimerization with other members of the EGFR family. Beyond that, unlike other HER receptors, HER3 has 14 tyrosines in the C-terminal tail; 6 of them are docking sites directly recruiting the p85 subunit of PI3K. As a consequence, HER3 is a key node in the activation of the PI3K/Akt pathway, promoting tumor cell survival, proliferation, and metastasis (19). Recent studies have highlighted HER3 as a fundamental signaling receptor involved in the establishment of malignancy, with several groups demonstrating that HER3 overexpression correlates with advanced disease stage and decreased overall survival (20). In addition, emerging evidence from studies of EGFR- and HER2driven cancers have shown that these cancers invariably become resistant to anti-HER therapies, indicating that HER3 mediates in their treatment failures (21). For example, it has been demonstrated that there is a strong association between sensitivity to tyrosine kinase inhibitors, including gefitinib and erlotinib, and
HER3 inactivation $(22,23)$. These observations suggest that upregulation of HER3 receptors may be an indication of poor prognosis and that agents targeting HER3 could potentially provide a novel approach toward the treatment of such cancers.

Because HER3 lacks enzymatic activity, there have been efforts to develop mAbs targeting the receptor ectodomain and inhibiting various mechanisms by which HER3 can become activated, with some of these newly developed mAbs already being in phase I and II clinical trials (i.e., seribantumab [MM121], patritumab [U3-1287], and LJM716) $(24,25)$. The preclinical findings that have been presented for these so far appear to be promising. For example, a study of MM-121 combined with the anti-EGFR mAb cetuximab in a mouse model of lung cancer clearly showed that resistance to cetuximab is due to HER3 activation and that blocking of HER3 signaling by adding MM-121 to the treatment combination led to a greater and more durable response (26). Moreover, LJM716 has shown significant growth inhibitory effects in HER2-positive cells and xenograft models when combined with trastuzumab or cetuximab (27).

However, the activity of HER3-selective drugs in humans has not been robustly demonstrated, and the overall data may be viewed as modest. Recently, U3-1287 was used in combination with erlotinib in non-small cell lung cancer patients who had progressed after at least one course of chemotherapy. The authors reported that the efficacy of the combination was encouraging, but there was no clear correlation between tumor response and HER3 expression in tumor tissues or serum soluble HER3 levels. The lack of correlation could be due to the

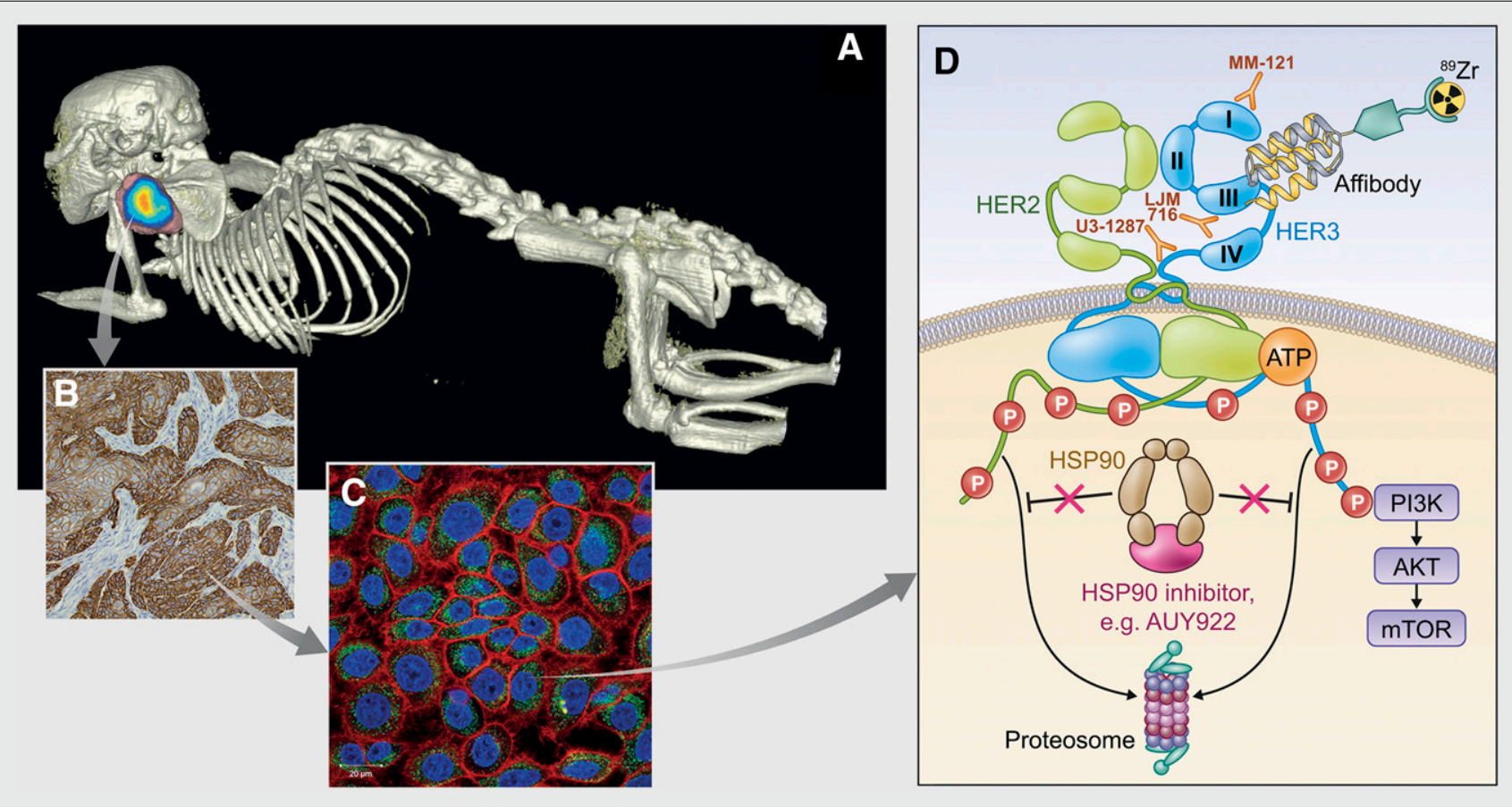

FIGURE 3. (A) ${ }^{89 Z r-A f f i b o d y}{ }_{\text {HER } 3: 8698}$ uptake $3 \mathrm{~h}$ after injection in mouse bearing MCF-7 tumor. (B) Ex vivo HER3 immunohistochemistry staining of MCF-7 tumor showing distribution of HER3 receptors at different tumor regions. (C) Confocal microscopy image of HER3-

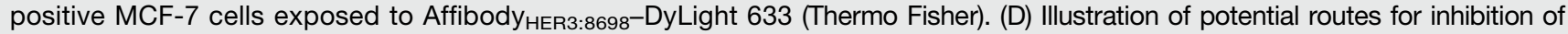
HER2/HER3 pathway activation and imaging of receptor status. ATP = adenosine triphosphate; HSP90 = heat shock protein 90 ; $\mathrm{mTOR}=$ mammalian target of rapamycin. 
type of tumor tissue that was used (archived tissue from the initial diagnosis) or the relatively low number of patients enrolled in the study (28). Therefore, the absence of a suitable biomarker to evaluate HER3 status still remains the major hurdle in the development of HER3-targeted therapies and effective patient selection. Consideration has been given to markers established for overexpression of partner kinases, such as HER2, or markers for the expression level of ligands that activate HER3, such as NRG-1. For example, Schoeberl et al. have shown that the level of NRG-1 can be a better biomarker than receptor expression for selection of patients who may benefit from MM-121 treatment (26). But even though NRG-1 is the most potent inducer of HER3 phosphorylation, using it as an effective biomarker might be quite limiting because of the existence of multiple routes of HER3 activation, such as mutation and ligand-independent dimerization, and the potential contribution to receptor activation from other HER family ligands.

In light of these findings, there is clearly a need to develop imaging biomarkers that can guide the clinical use of innovative anti-HER3-targeted therapies. So far, to assess the HER3 tumor status, ${ }^{89} \mathrm{Zr}$-radiolabeled mAb (GSK2849330) is being evaluated in patients with advanced solid tumors (clinical trial NCT12345174).

In addition, in a small-animal SPECT/CT study, a novel ${ }^{111} \mathrm{In}$ labeled bispecific agent consisting of a trastuzumab Fab fragment targeting HER2 and NRG-1 targeting HER3 has been shown to specifically accumulate in tumor xenografts in which HER2 is coexpressed with HER3 (29).

Recently, we have developed ${ }^{89}$ Zr-labeled Affibody ${ }_{\text {HER } 3: 8698}$, which recognizes the HER3 antigen (Fig. 3). High tracer accumulation was found in human breast cancer MCF-7 and BT-474 $($ HER3 +++$)$ xenografts but not in MDA-MB-231 tumors $(\mathrm{HER} 3+)$, confirming the binding specificity of the probe. Similar images showing high contrast between targeted and nontargeted tissues were obtained for ${ }^{89} \mathrm{Zr}$-RG7116, an anti-HER3 antibody (30), but $144 \mathrm{~h}$ were required for the mAb to be cleared, whereas the ${ }^{89} \mathrm{Zr}$-Affibody ${ }_{\text {HER 3:8698 }}$ provided images within 1-3 $\mathrm{h}$ after injection. Furthermore, when mice bearing MCF7 tumors were treated with NVP-AUY922, which is known to induce proteasome-mediated degradation of phosphorylated HER2 and HER3, there was a significantly higher tumor uptake of ${ }^{89} \mathrm{Zr}$-Affibody ${ }_{\text {HER 3:8698 }}$ in mice receiving the drug than in the control animals. This unexpected enhancement in HER3 expression after the treatment has been found to correlate with the recovery of HER3 and subsequent upregulation of the insulinlike growth factor 1 receptor and activation of the PI3K/Akt pathway (unpublished data). These data underline the potential that a HER3-imaging agent can serve as a tool not only for patient stratification for HER3-targeted therapies but also, more importantly, for monitoring receptor level during therapeutic interventions.

\section{CONSIDERATIONS AND FUTURE PERSPECTIVES}

The observation that HERs are key players in the establishment of malignancy have led to several mAbs and T-cell inhibitors blocking EGFR and HER2, resulting in clinically meaningful anticancer activity. Continuously growing evidence points to HER3 upregulation as an important factor in resistance to HER-targeted therapies. The number of anti-HER3 antibodies currently under development and tested in clinical trials further underscores the importance of this target. Experimental and clinical evidence suggests that the dynamic interactions of HER3 force the combination of agents that directly block HER3 with compounds that interfere with receptor dimer signaling. The use of specific imaging agents as cancer biomarkers could provide unique information, including quantification of receptor heterogeneity and early detection of changes in target expression in response to therapy in parallel to high-throughput sequencing technologies.

\section{DISCLOSURE}

No potential conflict of interest relevant to this article was reported.

\section{REFERENCES}

1. Arteaga CL, Engelman JA. ERBB receptors: from oncogene discovery to basic science to mechanism-based cancer therapeutics. Cancer Cell. 2014;25:282-303.

2. Yarden Y, Pines G. The ERBB network: at last, cancer therapy meets systems biology. Nat Rev Cancer. 2012;12:553-563.

3. Morgan P, Van Der Graaf PH, Arrowsmith J, et al. Can the flow of medicines be improved? Fundamental pharmacokinetic and pharmacological principles toward improving phase II survival. Drug Discov Today. 2012;17:419-424.

4. Dijkers EC, Oude Munnink TH, Kosterink JG, et al. Biodistribution of ${ }^{89} \mathrm{Zr}$ trastuzumab and PET imaging of HER2-positive lesions in patients with metastatic breast cancer. Clin Pharmacol Ther. 2010;87:586-592.

5. Tamura K, Kurihara H, Yonemori K, et al. ${ }^{64} \mathrm{Cu}$-DOTA-trastuzumab PET imaging in patients with HER2-positive breast cancer. J Nucl Med. 2013;54:1869-1875.

6. Smith-Jones PM, Solit D, Afroze F, Rosen N, Larson SM. Early tumor response to Hsp90 therapy using HER2 PET: comparison with ${ }^{18}$ F-FDG PET. J Nucl Med. 2006;47:793-796.

7. Beylergil V, Morris PG, Smith-Jones PM, et al. Pilot study of ${ }^{68}$ Ga-DOTA-F(ab')2trastuzumab in patients with breast cancer. Nucl Med Commun. 2013;34:1157-1165.

8. Gaykema SB, Schroder CP, Vitfell-Rasmussen J, et al. ${ }^{89} \mathrm{Zr}$-trastuzumab and ${ }^{89} \mathrm{Zr}$ bevacizumab PET to evaluate the effect of the HSP90 inhibitor NVP-AUY922 in metastatic breast cancer patients. Clin Cancer Res. 2014;20:3945-3954.

9. Gebhart G, Lamberts LE, Wimana Z, et al. Molecular imaging as a tool to investigate heterogeneity of advanced HER2-positive breast cancer and to predict patient outcome under trastuzumab emtansine (T-DM1): the ZEPHIR trial. Ann Oncol. 2016;27:619-624.

10. Kramer-Marek G, Bernardo M, Kiesewetter DO, et al. PET of HER2-positive pulmonary metastases with ${ }^{18} \mathrm{~F}$-ZHER2:342 Affibody in a murine model of breast cancer: comparison with ${ }^{18}$ F-FDG. $J$ Nucl Med. 2012;53:939-946.

11. Baum RP, Prasad V, Muller D, et al. Molecular imaging of HER2-expressing malignant tumors in breast cancer patients using synthetic ${ }^{111} \mathrm{In}$ - or ${ }^{68} \mathrm{Ga}$-labeled Affibody molecules. J Nucl Med. 2010;51:892-897.

12. Sörensen J, Sandberg D, Sandstrom M, et al. First-in-human molecular imaging of HER2 expression in breast cancer metastases using the ${ }^{111}$ In-ABY-025 Affibody molecule. J Nucl Med. 2014;55:730-735.

13. Aerts HJWL, Dubois L, Perk L, et al. Disparity between in vivo EGFR expression and ${ }^{89} \mathrm{Zr}$-labeled cetuximab uptake assessed with PET. J Nucl Med. 2009;50:123-131.

14. Pines G, Kostler WJ, Yarden Y. Oncogenic mutant forms of EGFR: lessons in signal transduction and targets for cancer therapy. FEBS Lett. 2010;584:2699-2706.

15. Chang AJ, De Silva RA, Lapi SE. Development and characterization of ${ }^{89} \mathrm{Zr}$ labeled panitumumab for immuno-positron emission tomographic imaging of the epidermal growth factor receptor. Mol Imaging. 2013;12:17-27.

16. van Dijk LK, Boerman OC, Franssen GM, Kaanders JH, Bussink J. ${ }^{111}$ In-cetuximab$\mathrm{F}(\mathrm{ab}) 2$ SPECT and ${ }^{18} \mathrm{~F}$-FDG PET for prediction and response monitoring of combined-modality treatment of human head and neck carcinomas in a mouse model. J Nucl Med. 2015;56:287-292.

17. Cai W, Niu G, Chen X. Multimodality imaging of the HER-kinase axis in cancer. Eur J Nucl Med Mol Imaging. 2008;35:186-208. 
18. Gelovani JG. Molecular imaging of epidermal growth factor receptor expressionactivity at the kinase level in tumors with positron emission tomography. Cancer Metastasis Rev. 2008;27:645-653.

19. Schoeberl B, Pace EA, Fitzgerald JB, et al. Therapeutically targeting ErbB3: a key node in ligand-induced activation of the ErbB receptor-PI3K axis. Sci Signal. 2009;2:ra31.

20. Friess H, Yamanaka Y, Kobrin MS, Do DA, Buchler MW, Korc M. Enhanced erbB-3 expression in human pancreatic cancer correlates with tumor progression. Clin Cancer Res. 1995;1:1413-1420.

21. Amin DN, Campbell MR, Moasser MM. The role of HER3, the unpretentious member of the HER family, in cancer biology and cancer therapeutics. Semin Cell Dev Biol. 2010;21:944-950.

22. Engelman JA, Janne PA, Mermel C, et al. ErbB-3 mediates phosphoinositide 3kinase activity in gefitinib-sensitive non-small cell lung cancer cell lines. Proc Natl Acad Sci USA. 2005;102:3788-3793.

23. Buck E, Eyzaguirre A, Haley JD, Gibson NW, Cagnoni P, Iwata KK. Inactivation of Akt by the epidermal growth factor receptor inhibitor erlotinib is mediated by HER-3 in pancreatic and colorectal tumor cell lines and contributes to erlotinib sensitivity. Mol Cancer Ther. 2006;5:2051-2059.

24. Aurisicchio L, Marra E, Roscilli G, Mancini R, Ciliberto G. The promise of antiErbB3 monoclonals as new cancer therapeutics. Oncotarget. 2012;3:744-758.
25. Garner AP, Bialucha CU, Sprague ER, et al. An antibody that locks HER3 in the inactive conformation inhibits tumor growth driven by HER2 or neuregulin. Cancer Res. 2013;73:6024-6035.

26. Schoeberl B, Faber AC, Li D, et al. An ErbB3 antibody, MM-121, is active in cancers with ligand-dependent activation. Cancer Res. 2010;70:2485-2494.

27. Garrett JT, Sutton CR, Kurupi R, et al. Combination of antibody that inhibits ligandindependent HER3 dimerization and a p110alpha inhibitor potently blocks PI3K signaling and growth of HER2+ breast cancers. Cancer Res. 2013;73:6013-6023.

28. Nishio M, Horiike A, Murakami H, et al. Phase I study of the HER3-targeted antibody patritumab (U3-1287) combined with erlotinib in Japanese patients with non-small cell lung cancer. Lung Cancer. 2015;88:275-281.

29. Razumienko EJ, Chen JC, Cai Z, Chan C, Reilly RM. Dual receptor-targeted radioimmunotherapy of human breast cancer xenografts in athymic mice coexpressing HER2 and EGFR using ${ }^{177} \mathrm{Lu}$ - or ${ }^{111}$ In-labeled bispecific radioimmunoconjugates. J Nucl Med. 2016;57:444-452.

30. Terwisscha van Scheltinga AG, Lub-de Hooge MN, Abiraj K, et al. ImmunoPET and biodistribution with human epidermal growth factor receptor 3 targeting antibody ${ }^{89} \mathrm{Zr}-\mathrm{RG} 7116$. MAbs. 2014;6:1051-1058. 